\title{
32. The Cosmic Dust Analyzer: Experimental Evaluation of an Impact Ionization Model
}

\author{
J. F. Friichtenicht, N. L. Roy, \\ AND D. G. BECKER \\ TRW Systems \\ Redondo Beach, California
}

\begin{abstract}
Determination of the elemental composition of cosmic dust particles by means of an impact ionization time-of-fight mass spectrometer has been investigated at several institutions. In most configurations, the instrument supplies the identity of ion groups of both target and particle materials extracted from the impact plasma and the number of ions contained in each group. Experiments have shown that the fractional ionization of a given species is not constant with impact velocity nor is the fractional ionization the same for different kinds of atoms. A model of the impact ionization effect developed at $T R W$ involves an equilibrium plasma condition with the consequence that the fractional ionization for an arbitrary atomic species can be specified by the Saha equation if the plasma volume (V) and temperature $(\mathrm{T})$ are known. It follows that $\mathrm{T}$ can be determined by taking the ratio of the Saha equations for two elements present in the target in known concentration. (Taking the ratio negates the requirement of knowing V.) Given T, the procedure can be reversed to yield the relative abundance of elements contained in the impacting particle. To test the model, a $\mathrm{PbZrO}_{3}-\mathrm{PbTiO}_{3}$ target was bombarded with high velocity $\mathrm{Fe}, \mathrm{MoB}$, and $\mathrm{NiAl}$ particles and the number of $\mathrm{Pb}, \mathrm{Ti}$, and $\mathrm{Zr}$ ions was determined in a time-of-flight mass spectrometer. For each event, the relative abundance of $\mathrm{T} i$ to $\mathrm{Pb}$ was taken as known (from electron microprobe analysis) and $\mathrm{T}$ was determined from the $\mathrm{T} i-\mathrm{Pb}$ measurement. The $\mathrm{Zr}$ to $\mathrm{Pb}$ ratio was found to be in good agreement with the microprobe analysis ( 0.38 calculated mean value compared to 0.34 actual). The result was valid for all particle materials and for a velocity range $17<\mathrm{v}<47 \mathrm{~km} / \mathrm{s}$. $\mathrm{T}$ ranged from 3800 to $11500^{\circ} \mathrm{K}$ and was only mildly velocity dependent.
\end{abstract}

$\mathrm{T}^{\mathrm{B}}$ HE COSMIC DUST ANALYZER under development at TRW may be described as an impact ionization time-of-flight mass spectrometer. As implied by this description, the instrument utilizes ion time-of-flight techniques to separate the microplasma formed by the impact of a high velocity particle upon a solid surface into its constituent parts. The plasma contains ions of both particle and target material, which may be extracted from the impact plasma by the applica- tion of an electric field to the impact surface. This is most easily accomplished by placing a potential difference between the impact surface and a high transmittance grid located in close proximity to the surface and parallel to it. All singly ionized atoms reach the plane of the grid with the same kinetic energy (given by the product of the unit electronic charge and the applied voltage); however, the velocity of a particular ion at this point depends upon its mass. Thus, the atomic mass of 
the ions may be determined by measuring their transit time from the grid to an ion collector located some distance away.

First, the formation of the plasma and the extraction of ions from it may be considered instantaneous, with the consequence that all ions of a given mass reach the collector at the same time. Actually, it has been observed that the ions may possess an appreciable thermal (random) velocity which adds to the electrically imparted velocity. This causes a small spread in arrival times of ions of a given charge-to-mass ratio. Nevertheless, by proper selection of accelerating voltage and drift distance, the ions can be segregated into distinct groups according to their mass. With the accelerating voltage and flight distance specified, measurement of the transit time is sufficient to determine the mass of each ion group. Determination of the atomic mass identifies the corresponding element.

The foregoing defines the basic instrumental requirements of the cosmic dust analyzer. The key measurements to be made are the transit time of the various ion groups from the impact surface to the ion collector and the total charge contained within each group. The instant of impact is signaled by the emission of positive charge from the impact (target) surface, resulting in a negative signal. This signal may be used to initiate the ion time-of-flight measurement. A convenient means of measuring the total charge within each ion group is to integrate the ion current at the collector. In this case the ion collector signal has a stairstep appearance; the position in time of a step corresponds to the time of arrival of a given ion group and its amplitude is proportional to the charge contained within the ion group. The number of ions in each group is obtained by dividing the total charge by the unit electron charge.

According to its description, the instrument supplies direct measurements of the number of ions in each group reaching the collector and provides the timing information necessary to identify the species of the ions. These data alone would be extremely useful in evaluating the gross characteristics of cosmic dust particles. However, a more sophisticated objective is that of quantitatively determining the relative abundances of the neutral elements contained in the particles. As might be expected intuitively and as has been verified experimentally, the fractional degree of ionization of all atomic species is not the same. Moreover, the relative ionizing efficiencies change with impact velocity. Hence, additional information is needed in order to process the raw data to obtain the desired final result. A method has been conceived to obtain that information; this paper will report the results of laboratory experiments which indicate that the method is a valid approach to the problem.

At the heart of the method is a theoretical model of the impact ionization process that was originally developed because it successfully explained various phenomena observed in earlier impact experiments. Briefly, the model assumes that the impact produces a plasma in thermal equilibrium. If this is the case and if the plasma temperature and volume can be found, the degree of ionization of any atomic species in the plasma can be computed provided that the species has a known ionization potential. The requirement to specify the plasma volume can be shown to disappear if we are content to determine relative abundances, leaving only the plasma temperature to be measured. If the impact target contains two elements in known concentration, the model provides equations by which measurement of the relative ion signals of these two elements can be reduced to plasma temperature. Given the temperature, the model supplies further theoretical relationships for processing the data to yield the relative atomic abundances of the elemental constituents in the impacting particle.

This model of impact ionization and the theoretical relationships derived therefrom should be experimentally verified. The best approach to this task appears to be to employ test particles and an impact target that are each composed of two elements in known relative concentrations, utilizing the theory to be described in the section of this paper on the impact ionization model to independently determine the plasma temperature from each of the two-element combinations. If the two values of plasma temperature so determined should agree for each hypervelocity impact event, then the model may be considered to have been verified. Unfortunately, we have been unable to locate a source of particles with sufficiently homogeneous properties to successfully complete this type of experiment. 
We have therefore developed an alternative approach that seems to be almost as satisfactory. The alternate involves the use of a target material containing three major elements with known relative abundances. Two of these are treated as control elements, and the relative ion abundances of the control species are observed and used to compute the plasma temperature as previously described. This plasma temperature and the magnitude of the ion signal from the third element are then combined to compute the relative atomic abundance of the latter. The computed value can of course be compared directly to the known abundance of the third material; good agreement indicates that the model is essentially correct. In comparison with the postulated "best" approach, the alternate suffers by not directly involving the properties of the impacting particle in the calculations, and the verification provided thereby assumes that the target and particle materials become intimately mixed in the impact plasma. This assumption requires its own experimental test. Even in the absence of such a test, however, any agreement found from the alternate experiment is strong evidence that the model is a reasonably good representation of the impact ionization phenomenon.

We will describe herein an experiment in which the alternative approach was employed and in which data were acquired for a number of impact events involving different particle compositions. As we hope to show, these data appear to possess a sufficiently good agreement with the known target composition that the desired verification can be said to have been achieved.

\section{IMPACT IONIZATION MODEL}

It was first shown experimentally by Friichtenicht and Slattery (1963) that free electric charge, both positive and negative, is produced at the point of impact of a high velocity microparticle on a solid surface. More recent experiments reported by Hansen (1968) and by Auer and Sitte (1968) have shown that the positive charge consists of ions of both the impacting particle and target materials and that the negative charge carriers are electrons. Unpublished data obtained at TRW have demonstrated this effect in considerable detail for a large number of particle-target material combinations. For low impact velocities, both Hansen and Auer and Sitte found that easily ionized impurities (sodium and potassium) accounted for the majority of the emitted positive charge even though the impacting particles were $\sim 98$ percent iron. At higher impact velocities, however, iron became the dominant ionic species, and ions of other high ionization potential elements made an appearance. Particularly significant was the detection of ions from refractory metal targets.

Based on these observations, Hansen (1968) advanced a theoretical model to describe the impact ionization effect. In this model, it is assumed that a small, high density plasma is formed in the immediate vicinity of the impact site. The plasma expands due to its internal pressure; however, the collision frequency of atoms and electrons within the plasma is very high (on the order of $10^{14}$ per second initially), with the consequence that the plasma temperature is spatially uniform (i.e., that the plasma temperature is nearly the same throughout the plasma volume) but decreases as the plasma expands. At some point the plasma becomes collisionless as the density decreases. To the extent that the plasma may be considered to be in thermal equilibrium immediately prior to becoming collisionless, the Hansen model described in the following paragraphs should apply.

When a plasma in thermal equilibrium at absolute temperature $T$ contains several species of atoms and ions, the degree of ionization of each species $s$ whose ionization energy is $E_{I}{ }^{s}$ is related to $T$ according to Saha's equation (cf. Sutton and Sherman, 1965) :

$$
n_{e} n_{i}^{\varepsilon} / n_{n}^{8}=C T^{3 / 2} u_{i, n^{8}} \exp \left(-E_{I^{2}} / k T\right)
$$

where $n_{e}, n_{i}{ }^{s}$, and $n_{n}{ }^{s}$ are the numbers per unit volume of electrons, ions of species $s$, and neutral atoms of species $s$, respectively; $C$ is a constant; $u_{i, n}{ }^{8}$ is the ratio $u_{i}^{8} / u_{n}^{8}$, in which $u_{i}^{8}$ and $u_{n}{ }^{8}$ are respectively the internal partition functions of ions and neutrals of species $s$; and $k$ is Boltzmann's constant. The Saha equation shows that the fractional ionization of a given species of atom depends on the ratio of the ionization energy of that species to the mean thermal energy of the plasma. For reasonably low plasma temperatures, only a small fraction of the atoms are ionized 
(except for those with very low ionization potentials). In order to use the impact ionization effect to reliably determine the relative abundance of the elements in cosmic dust and meteoroids, a measure of the plasma temperature must be obtained.

It would at first appear that the number density of ions, electrons, and atoms must be specified. However, from the argument given above, the plasma rapidly expands to a collisionless state. Thus, the total number of ions is constant at later times during the expansion process. Since all of the ions regardless of species are extracted from the same volume, a direct measurement of $n_{e}$, $n_{n}^{s}$, and $n_{i}^{s}$ is not required to determine the relative abundance of the different kinds of atoms. If $N_{e}, N_{n}^{s}$, and $N_{i}^{s}$ are, respectively, the total numbers of electrons, atoms, and ions in the plasma, and if $V$ is the volume of the plasma at any time prior to its expansion to a collisionless state, then $n_{e}=N_{e} / V, n_{n}^{s}=N_{n}^{s} / V$, and $n_{i}^{s}=N_{i}^{s} / V$. Clearly, $n_{e} n_{i}^{s} / n_{n}{ }^{s}$ can be replaced in equation (1) by $N_{e} N_{\imath}^{8} / N_{n}{ }^{8} V$. As we will shortly see, $V$ can be ignored in the type of experiment that we perform. Our experimental techniques provide a direct measurement of $N_{i}^{s}$ for each $s$ and of $N_{e}$. (If all $N_{i}{ }^{s}$ are measured, $N_{e}$ can be obtained as $\Sigma_{8} N_{i}{ }^{s}$, since the total number of electrons is equal to that of ions; however, an independent measurement of $N_{e}$ is readily obtained and serves as a useful check of the data.) The quantity of principal interest to be extracted from the experiments is $N_{n}{ }^{s}$.

Consider first an idealized case in which the plasma contains two species from the target and one from the particle; let these species be denoted by superscripts (1), (2), and (3), and assume that the identities of (1) and (2), their ionization energies $E_{I}{ }^{(1)}$ and $E_{I}{ }^{(2)}$, and their partition function ratios $u_{i, n}{ }^{(1)}$ and $u_{i, n}{ }^{(2)}$ are all known. If equation (1) is written for species (1) and for species (2) and if the former result is divided by the latter, one finds that

$$
\begin{aligned}
&\left(N_{i}^{(1)} / N_{i}^{(2)}\right)\left(N_{n}^{(2)} / N_{n}^{(1)}\right) \\
&=f^{\prime}(T) \exp \left[\left(E_{I}^{(2)}-E_{I}^{(1)}\right) / k T\right]
\end{aligned}
$$

in which $f^{\prime}(T) \equiv u_{i, n}{ }^{(1)} / u_{i, n}{ }^{(2)}$. (The partition functions are functions of temperature; the functional dependence is assumed to be known.) It is almost always true that $N_{n}^{s} \gg N_{i}^{s}$, and then the ratio $N_{n}{ }^{(1)} / N_{n}{ }^{(2)}$ is approximately equal to the relative concentration of the two kinds of atoms in the target material. Let $A$ denote this ratio, whose value is known. Now, suppose that species (1) and (2) have been selected such that $E_{I}^{(2)} \neq$ $E_{I}^{(1)}$. Writing $E_{I}^{(2)}=E_{I}^{(1)}+\Delta E_{I}^{(1,2)}$ and substituting into equation (2), we obtain

$$
N_{i}^{(1)} / N_{i}{ }^{(2)}=f^{\prime}(T) A \exp \left(-\Delta E_{I}^{(1,2)} / k T\right)
$$

from which

$$
k T=\Delta E_{I}^{(1,2)}\left\{\ln \left[f^{\prime}(T) A\left(N_{i}^{(2)} / N_{i}^{(1)}\right)\right]\right\}^{-1}
$$

According to equation (4), the plasma temperature can be found from a measurement of the number of ions of the two control elements in the target. (Remember that $f^{\prime}(T)$ is a known function.) If, as often happens, $f^{\prime}(T)$ is essentially constant over a range of temperatures, the computation of $T$ from the $N_{i}{ }^{(2)} / N_{i}{ }^{(1)}$ measurement is quite simple; if $f^{\prime}(T)$ is not constant, iterative solutions can be employed.

It follows at once that the procedure employed to derive equation (2) can be applied for species (1) and (3), with the result:

$$
\begin{aligned}
N_{n}{ }^{(3)} / N_{n}{ }^{(1)}=g^{\prime}(T) & \left(N_{i}^{(3)} / N_{i}^{(1)}\right) \\
& \times \exp \left[\left(E_{I}^{(3)}-E_{I}^{(1)}\right) / k T\right]
\end{aligned}
$$

where $g^{\prime}(T) \equiv u_{i, n}{ }^{(1)} / u_{i, n}{ }^{(3)}$. Thus, if $N_{i}{ }^{(3)} / N_{i}{ }^{(1)}$ has also been measured, the relative abundance $N_{n}{ }^{(3)} / N_{n}{ }^{(1)}$ can be computed. The procedure is readily generalized to a situation in which the particle contains a multiplicity of elements and the relative abundance of each is computed in turn from measurements of the corresponding ion ratios. This is the way in which it is intended to reduce data from the actual cosmic dust analyzer experiment.

We saw that one way of checking the validity of these equations in this application would be to employ not only a target containing two control species (1) and (2), but also a test particle containing two other species whose relative concentrations in the particle are known; these are species (3) and (4). If the impact ionization model is valid, an equation similar to equation (4) can be written for the latter species, replacing $N_{i}^{(2)} / N_{i}^{(1)}$ by $N_{i}^{(4)} / N_{i}^{(3)}, f^{\prime}(T)$ by $h^{\prime}(T) \equiv$ $u_{i, n}{ }^{(3)} / u_{i, n}{ }^{(4)}$, and $\Delta E_{I}^{(1,2)}$ by $\Delta E_{I^{(3,4)}}$. Then a measurement of $N_{i}{ }^{(4)} / N_{i}{ }^{(3)}$ would give an independent determination of $T$ that could be compared with that found from $N_{i}{ }^{(2)} / N_{i}{ }^{(1)}$; agree- 
ment would strongly imply that the predictions of the model are correct.

The alternative check, used as the basis of our experiment for the reasons stated earlier, consists of employing a three-constituent target, with the relative abundances of all constituents known. We argue that the equilibrium plasma model implies a thorough mixing of the plasma constituents and that, as a result, target and particle constituents are interchangeable in the equations. Denoting the target species by (1), (2), and (3), we treat (1) and (2) as "knowns," using a $N_{i}^{(1)} / N_{i}{ }^{(2)}$ measurement to compute $T$ according to equation (4), and (3) as the "unknown," computing $N_{n}^{\left({ }^{(3)}\right.} / N_{n}{ }^{(1)}$ from the temperature calculation and equation (5). Of course, the actual value of $N_{n}{ }^{(3)} / N_{n}{ }^{(1)}$ is known; this known value can be compared to that computed from equation (5), and good agreement is evidence for the validity of the model. We noted that the evidence is not quite so strong as in the first approach, because now the particle properties do not directly enter into the calculations and hence the assumption of mixing, which is central to this work, remains an assumption.

In spite of the above limitation, it is important to observe that the state of knowledge of the impact ionization effect with respect to basic theoretical considerations is sufficiently primitive at the present time that any direct evidence for the validity of a proposed theoretical model of the process would represent a major advance. This was an important consideration in our decision to perform the experiment to be described.

\section{EXPERIMENTAL APPARATUS AND TECHNIQUES}

All of the experimental data on the impact ionization effect were acquired with the TRW electrostatic microparticle accelerator described by Friichtenicht (1962). This device is a two million volt Van de Graaff generator in which the high voltage terminal has been modified to accept microparticle charging and injection equipment. It is capable of charging and accelerating a variety of micron size range particulate materials to high velocities. The final velocity obtained is dependent upon the size of the particular particle, the material used, and the accelerating potential.
Particle velocities range down to about $1 \mathrm{~km} / \mathrm{s}$ for all materials. The upper velocity limit is, for practical purposes, dependent upon the electronics used for particle detection. For carbonyl iron particles, the maximum detectable velocity is about $50 \mathrm{~km} / \mathrm{s}$; for lower density materials, the maximum velocity is much higher. Particles may be accelerated either continually or in single bursts.

Particles from the accelerator pass through a particle charge-velocity detector which consists of a cylindrical drift tube of known length and capacitance and an associated amplifier. The rectangular pulse derived from this detector as the charged particle traverses jts length provides the necessary data for computing particle mass and velocity. The details have been described elsewhere (Shelton et al., 1960; Hansen and Roy, 1966).

The charged particle "beam" from the accelerator has a broad distribution due to particle size variation. For experimental convenience the beam is further conditioned by means of particle velocity (i.e., transit time) selection equipment (Roy and Becker, 1971) which may be set to limit the particles reaching the experimental area to only those having a velocity lying within a narrow velocity range. All others are electrically deflected.

A detailed block diagram of the experimental apparatus including a schematic representation of the target and collector assemblies is shown in figure 1 . The target and collector assemblies are housed in a cylindrical vacuum enclosure about $45 \mathrm{~cm}$ in diameter by $125 \mathrm{~cm}$ long. Particles from the accelerator traverse a velocity selection system (not shown in the figure) and then a charged particle detector prior to entry into the main experimental volume. The particles pass through an aperture in the ion collector and proceed onward to impact on the target assembly.

The target assembly is mounted at the downstream end of the system (most distant from the accelerator) such that simulated micrometeoroids produced by the accelerator will impact near the center of the target and normal to its surface. Since particles from the accelerator are confined to a $6.3 \mathrm{~mm}$ diameter circle, a $5 \mathrm{~cm}$ diameter target was considered ample for these tests. The target is mounted inside a shielded housing as 

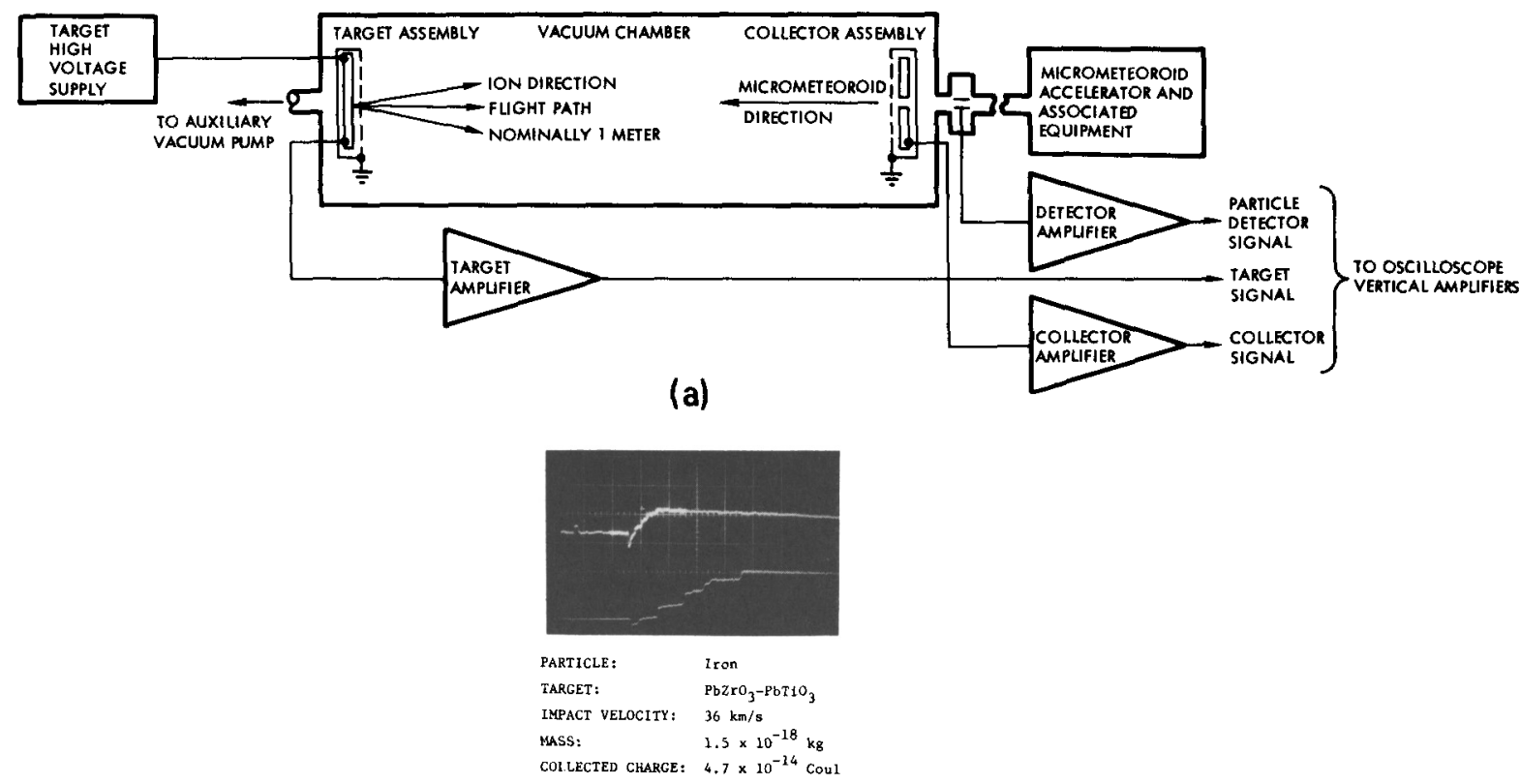

(b)

Figure 1.-Detailed block diagram of the experimental configuration, with the internal target and collector assemblies depicted schematically. Signal outputs from the experiment are illustrated in (b); the upper trace is the sum of particle detector, target, and collector signals with sweep speed set at $20 \mu \mathrm{sec} / \mathrm{div}$; lower trace, target and collector signals at $5 \mu \mathrm{sec} / \mathrm{div}$.

shown in the figure, with the extraction and acceleration grid mounted approximately $1 \mathrm{~cm}$ from the target surface. Cable connectors with hermetic seals provide means for introducing the target bias voltage $(+3000 \mathrm{~V})$ and withdrawing the target signal from the vacuum chamber.

The ion collector mechanical assembly is mounted at the opposite (upstream) end of the vacuum chamber at a distance of approximately $1 \mathrm{~m}$ from the target. The collector plate is about $30 \mathrm{~cm}$ in diameter and is centered on the axis of the particle beam. The entire assembly has a small hole (about $1.25 \mathrm{~cm}$ ) at the center which allows the particles to pass. The collector plate is surrounded by a grounded shield. In order to maintain electrical shielding while permitting ions to enter, the side of the shield which faces the target is made from screening cloth of reasonably high transmittance. The large diameter collector plate is required to collect a substantial fraction of the ions originating at the target, due to expansion of the beam as a result of the initial thermal energy of the ions. Again, a hermetically sealed cable connector allows the collector signal to be extracted from the vacuum enclosure.

The photographic record of a typical event is shown in figure 1 (b). This particular oscillogram records the data from the impact of an iron particle on a $\mathrm{PbZrO}_{3}-\mathrm{PbTiO}_{3}$ target at $36 \mathrm{~km} / \mathrm{s}$. The sequence of events begins when a particle from the accelerator is found by the time interval selection equipment to have a transit time within the preselected transit time (velocity) boundaries. This event causes the bias voltage on a particle deflector to be removed, thereby allowing the selected particle to enter the experimental region. Just prior to arrival of the particle at the particle detector depicted schematically in figure 1, a proportional delay generator produces an output pulse to initiate an oscilloscope sweep that will present the signal amplitude and transit time information produced by the particle as it passes through the detector (upper trace on the oscillogram). This information allows the particle parameters (velocity, mass, and radius) to be calculated for each impacting particle. The particle then passes through the collector aperture 
enroute to the target. Just prior to arrival of the particle at the target, a second sweep is initiated which will present the target impact signal and the ion charge and transit time information (lower trace of the oscillogram). The signal obtained from the target is essentially a step function for impacts above about $10 \mathrm{~km} / \mathrm{s}$, while that from the collector rises in staircase fashion. Either or both waveforms may be differentiated to produce sharp pulses at impact and/or upon arrival of each ion group at the collector.

In Figure 1(b), the lower trace is the sum of the target and collector signals at a sweep speed of $5 \mu \mathrm{sec} /$ division. The initial negative step represents charge leaving the target, while the following positive-going steps represent the same charge arriving at the collector, now separated into individual charge "packets" distinguishable as ions of the constituent elements of particle and target material. The upper trace is the sum of the signals from the particle detector, the target, and the collector. The upper trace is presented at a slower sweep speed (20 $\mu \mathrm{sec} /$ division), insuring that the waveform being measured is due to the impact of the detected particle.

A total of 33 separate impact events on a lead titanate-lead zirconate target were recorded using the apparatus shown in figure 1. Of these, 12 particles were nickel-aluminide, seven were molybdenum boride, and the remainder were iron. The impact velocity range extended from 16.8 to $46.8 \mathrm{~km} / \mathrm{s}$. For each event the total number of lead, zirconium, and titanium ions reaching the collector was measured.

\section{DATA ANALYSIS}

All data from impacts of $\mathrm{Fe}, \mathrm{MoB}$, and $\mathrm{NiAl}$ particles onto the $\mathrm{PbZrO}_{3} / \mathrm{PbTiO}_{3}$ target sample were analyzed in accordance with the equilibrium plasma model discussed in the section on the impact model. There we saw that two of the target elements were to be treated as knowns and used to compute the relative abundances of the third element treated as an unknown. The ionization energies of $\mathrm{Pb}, \mathrm{Ti}$, and $\mathrm{Zr}$ are, respectively, $7.42 \mathrm{eV}, 6.82 \mathrm{eV}$, and $6.84 \mathrm{eV}$. Examination of equation (3) shows that the two control species (1) and (2) must have an appreciable difference $\Delta E_{1}{ }^{(1,2)}$ in ionization energy in order for this equation to yield meaningful results. This clearly required that $\mathrm{Pb}$ be one of the control elements. $\mathrm{Ti}$ was arbitrarily selected as the second control element, meaning that $\mathrm{Zr}$ became the "unknown."

The appropriate partition functions for all three elements were available in the literature (Aller, 1963) at four fixed temperatures: 3600 , 5700,8000 , and $10000^{\circ} \mathrm{K}$. Using these values and the ionization energies of the elements, we were able to compute values of the functions

$$
f(T) \equiv f^{\prime}(T) \exp \left(-\Delta E_{I}^{(1,2)} / k T\right)
$$

and

$$
g(T) \equiv g^{\prime}(T) \exp \left[\left(E_{I}^{(3)}-E_{I}^{(1)}\right) / k T\right]
$$

where superscripts (1), (2), and (3) now signify $\mathrm{Pb}, \mathrm{Ti}$, and $\mathrm{Zr}$, at the four given temperatures. On the assumption that the partition function ratios are smooth, well-behaved functions, we used the computations to $\operatorname{plot} f(T)$ and $g(T)$ vs $T$. Equations (3) and (5) can be rewritten

$$
N_{i}^{(1)} / N_{i}^{(2)}=A f(T)
$$

and

$$
N_{n}{ }^{(3)} / N_{n}{ }^{(1)}=\left(N_{i}^{(3)} / N_{i}{ }^{(1)}\right) g(T)
$$

The experiment gave measured values of $N_{i}(\mathrm{~Pb}) / N_{i}(\mathrm{Ti})$, which equals $N_{i}^{(1)} / N_{i}^{(2)}$, and $N_{i}(\mathrm{Zr}) / N_{i}(\mathrm{~Pb})$, which equals $N_{i}{ }^{(3)} / N_{i}{ }^{(1)}$. Since $A$ is given by $N_{n}(\mathrm{~Pb}) / N_{n}(\mathrm{Ti})$ and is known, the former value could be used to compute $f(T)$. From the plots, $T$ and then $g(T)$ were found, after which $N_{n}(\mathrm{Zr}) / N_{n}(\mathrm{~Pb})$ could be evaluated.

The "true" relative abundances of $\mathrm{Pb}, \mathrm{Ti}$, and $\mathrm{Zr}$ in the target sample were determined by electron microprobe analyses. To check for inhomogeneities, readings were taken at four separate points on the impact surface using an incident electron beam diameter on the order of one micron, with an analyzed depth also on the order of 1 micron. The composition found for the four points (table 1) indicates good homogeneity. The mean composition of the crystal was found to be as follows:

$$
\begin{aligned}
\mathrm{Pb} & =58.62 \pm .55 \text { atomic percent } \\
\mathrm{Ti} & =21.17 \pm .16 \text { atomic percent } \\
\mathrm{Zr} & =19.73 \pm .67 \text { atomic percent }
\end{aligned}
$$

where all quoted error values are $2 \sigma$ limits. In 
terms of relative abundances,

and

$$
N_{n}(\mathrm{Ti}) / N_{n}(\mathrm{~Pb})=0.361 \pm .006
$$

$$
N_{n}(\mathrm{Zr}) / N_{n}(\mathrm{~Pb})=0.337 \pm .015
$$

The mechanics of data reduction to $N_{n}(\mathrm{Zr}) /$ $N_{n}(\mathrm{~Pb})$ were straightforward and should be obvious in the light of the preceding discussion. The graphical method by which $f(T)$ values were used to obtain $g(T)$ naturally contains a number of approximations whose effects on the accuracy of the experiment are difficult to determine. Existing theory is sufficiently complete to permit the use of a high-speed digital computer to separately evaluate the partition functions $u_{i}$ and $u_{n}$ for almost any material at any temperature. Ideally, such a computation could have been performed for a large number of temperatures, after which the computer could have evaluated
$f(T)$ and $g(T)$ at these temperatures. Interpolations necessary to obtain the functions at any temperature could also have been included in the program. Such techniques will actually be employed to reduce cosmic dust analyzer in the space experiment, but they did not appear justified in the present context due to time and cost factors. After examining all aspects of the current experiment, we conclude that our graphical approxi-

Table 1.-Electron Microprobe Analysis of Target Sample

\begin{tabular}{lcccc}
\hline \multicolumn{1}{c}{ Element } & Point & Point & Point & Point \\
& 1 & 2 & 3 & 4 \\
\hline $\mathrm{Pb}$ (atomic percent) & 59.08 & 57.90 & 59.00 & 58.48 \\
$\mathrm{Ti}$ (atomic percent) & 21.24 & $\mathbf{2 1 . 1 9}$ & $\mathbf{2 1 . 9 9}$ & 20.95 \\
$\mathrm{Zr}$ (atomic percent) & 19.68 & 18.93 & 19.72 & 20.57
\end{tabular}

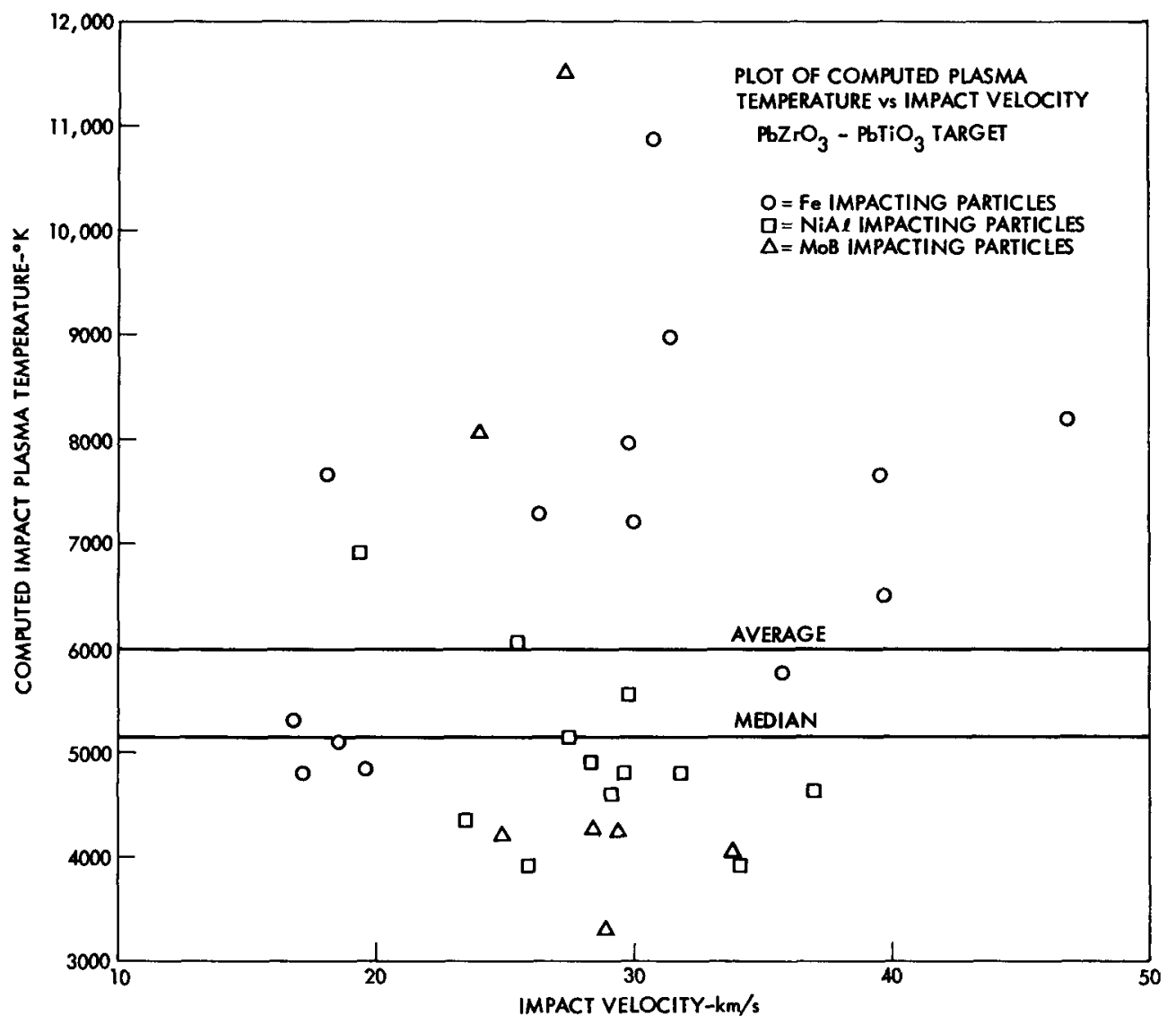

Figure 2.-Computed impact plasma temperature vs particle impact velocity: various particles, $\mathrm{PbZrO}_{3}-\mathrm{PbTiO}_{3}$ target. Data points shown are the computed result using the known relative abundance of two target elements together with the impact ionization model. Computed average and median impact temperatures as indicated. 
mation has not materially affected its significance or validity.

Figure 2 is a plot of the impact plasma temperature computed from equations (7a) and (4) as a function of impact velocity. The data scatter widely and there is no observable trend with increasing velocity; $\mathrm{NiAl}$ impacts appear to produce lower plasma temperatures on the average than do Fe impacts. (The model would predict such a trend due to the lower ionization potential of aluminum.) These results are similar to what has been observed with all other particle/target combinations employed during this program, and they tend to confirm that mechanical details of the materials at the impact sites (yield strengths, angle of impact, hardness, etc.), all of which are essentially random variables in the size range of the particles impacted, are more important in determining the amount of energy available for material vaporization and ionization than is the total energy of impact.

Remember, however, that the equilibrium plasma model says nothing at all regarding the relationship, if any, between impact velocity and plasma temperature. In fact, when the seemingly scattered data are analyzed further to compute $N_{n}(\mathrm{Zr}) / N_{n}(\mathrm{~Pb})$ according to equation (7b), surprisingly good agreement is found from one point to the next. Table 2 summarizes the velocity, plasma temperature, and relative $\mathrm{Zr}$ abundance $N_{n}(\mathrm{Zr}) / N_{n}(\mathrm{~Pb})$ for all of the data; the average $\mathrm{Zr}$ abundance is

$$
<N_{n}(\mathrm{Zr}) / N_{n}(\mathrm{~Pb})>=0.38 \pm .12
$$

where the error value is a $2 \sigma$ limit. Comparison with equation (8b) shows very good agreement, and the value of the standard deviation $(\sigma=0.06)$ for the 33 points is reasonably small. In addition, no one data point deviates more than about 40 percent from the average, which is rather remarkable given the fact that the data were acquired over a considerable period of time with three different particle materials and the method of analysis was rather crude in several aspects, as we have noted.

The sensitivity of this system for predicting elemental relative abundances from ion ratio measurements is illustrated in figure 3 , where we have plotted the $\mathrm{Zr} / \mathrm{Pb}$ ion ratio as a function of the $\mathrm{Ti} / \mathrm{Pb}$ ion ratio. The three curves have been computed for different assumed compositions of the target material, using the four fixed temperatures at which the partition functions were available; the middle curve corresponds to the actual composition given by the microprobe analysis, the upper curve to a 50 percent higher $\mathrm{Zr}$ content, and the lower curve to a 50 percent lower $\mathrm{Zr}$ content. (In both cases, the Ti content has been altered in inverse proportion.) The measured ion ratios are seen to cluster quite closely to the middle curve, and there is little difficulty in determining that the $\mathbf{Z r}$ content of the substance is much closer to 20 percent than to either 10 or 30 percent.

Figure 4 is a plot of the computed $\mathrm{Zr} / \mathrm{Pb}$ ratio as a function of impact velocity. It is included as one possible check on the validity of the model and the method of analysis, since if both are correct the ratio $N_{n}(\mathrm{Zr}) / N_{n}(\mathrm{~Pb})$ should be independent of $v$. The plot shows this to be the case.

\section{CONCLUSIONS}

The results presented in the preceding section provide fairly convincing proof that the use of an assumed thermal equilibrium plasma model can be used to process data from an impact ionization time-of-flight mass spectrometer in order to convert the raw ion data to relative abundances of the elemental constituents of cosmic dust particles. We do not wish to imply that the equilibrium condition is actually met; even intuitive arguments would suggest that a plasma cannot be in thermal equilibrium shortly before the plasma becomes collisionless, "because the collision frequency must approach zero in some asymptotic fashion. However, a "quasi-equilibrium" plasma is conceivable, and the analysis would then yield an "effective" plasma temperature. On the basis of the data presented in figure 3 , the model appears to be relatively sensitive in computing relative abundances. Considering the application and the precision requirements that can sensibly be placed on this kind of flight experiment, the rudimentary model appears to be quite satisfactory.

As mentioned earlier, a remaining question is verification that the particle material becomes thoroughly mixed with the target material, which is a requirement if a common plasma temperature is to be assigned to both materials. Our search for 
TABLE 2.-Summary of Experimental Data

\begin{tabular}{|c|c|c|c|c|}
\hline $\begin{array}{c}\text { Date and } \\
\text { particle material }\end{array}$ & $\begin{array}{c}\text { Temperature } \\
\left({ }^{\circ} \mathrm{K}\right)\end{array}$ & $\begin{array}{l}\text { Velocity } \\
(\mathrm{km} / \mathrm{s})\end{array}$ & $N_{n}(\mathrm{Zr}) / N_{n}(\mathrm{~Pb})$ & Squared deviation \\
\hline $\begin{array}{l}9 / 17 / 70 \\
\text { MoB particles }\end{array}$ & $\begin{array}{r}4480 \\
4200 \\
11500 \\
8050 \\
4050 \\
3300 \\
4270\end{array}$ & $\begin{array}{l}29.4 \\
24.8 \\
27.3 \\
24.0 \\
33.8 \\
28.9 \\
28.4\end{array}$ & $\begin{array}{l}0.255 \\
0.354 \\
0.374 \\
0.408 \\
0.375 \\
0.250 \\
0.265\end{array}$ & $\begin{array}{l}0.01440 \\
0.00044 \\
0.00000 \\
0.00109 \\
0.00000 \\
0.01562 \\
0.01210\end{array}$ \\
\hline $\begin{array}{l}9 / 22 / 70 \\
\text { NiAl particles }\end{array}$ & $\begin{array}{l}5550 \\
4350 \\
6900 \\
5150 \\
3925 \\
4600 \\
4800 \\
4630 \\
4800 \\
6050 \\
4900 \\
3920\end{array}$ & $\begin{array}{l}29.8 \\
23.4 \\
19.4 \\
27.5 \\
34.1 \\
29.3 \\
31.8 \\
37.0 \\
29.6 \\
25.5 \\
28.3 \\
25.9\end{array}$ & $\begin{array}{l}0.423 \\
0.295 \\
0.239 \\
0.419 \\
0.371 \\
0.393 \\
0.403 \\
0.374 \\
0.444 \\
0.323 \\
0.384 \\
0.357\end{array}$ & $\begin{array}{l}0.00230 \\
0.00640 \\
0.01850 \\
0.00194 \\
0.00002 \\
0.00032 \\
0.00078 \\
0.00000 \\
0.00476 \\
0.00270 \\
0.00008 \\
0.00032\end{array}$ \\
\hline $\begin{array}{l}1 / 16 / 71 \\
\text { Fe particles }\end{array}$ & $\begin{array}{l}4850 \\
5100 \\
7650 \\
5300 \\
4800\end{array}$ & $\begin{array}{l}19.6 \\
18.5 \\
18.1 \\
16.8 \\
17.2\end{array}$ & $\begin{array}{l}0.314 \\
0.373 \\
0.419 \\
0.375 \\
0.391\end{array}$ & $\begin{array}{l}0.00372 \\
0.00000 \\
0.00194 \\
0.00000 \\
0.00026\end{array}$ \\
\hline \multirow[t]{2}{*}{$\begin{array}{l}3 / 4 / 71 \\
\text { Fe particles }\end{array}$} & $\begin{array}{r}7650 \\
7280 \\
8180 \\
7950 \\
6470 \\
7200 \\
8970 \\
10870 \\
5750\end{array}$ & $\begin{array}{l}39.5 \\
26.3 \\
46.8 \\
29.8 \\
29.7 \\
30.0 \\
31.4 \\
30.7 \\
35.7\end{array}$ & $\begin{array}{l}0.534 \\
0.401 \\
0.394 \\
0.391 \\
0.367 \\
0.432 \\
0.449 \\
0.430 \\
0.406\end{array}$ & $\begin{array}{l}0.02528 \\
0.00068 \\
0.00036 \\
0.00026 \\
0.00006 \\
0.00325 \\
0.00548 \\
0.00302 \\
0.00096\end{array}$ \\
\hline & & & $\mathrm{Av}=0.375$ & Sum $=0.12705$ \\
\hline
\end{tabular}

Figure 4.-Computed zirconium/lead relative abundance $N_{n}(\mathrm{Zr}) / N_{n}(\mathrm{~Pb})$ vs particle impact velocity. Zirconium/lead relative abundance is computed using measured experimental values and the impact ionization model. Each computed value for relative abundance is plotted against the particle impact velocity from which the experimental values were obtained. Target surface analysis, as determined by electron microprobe, is as indicated to the right of the figure. 


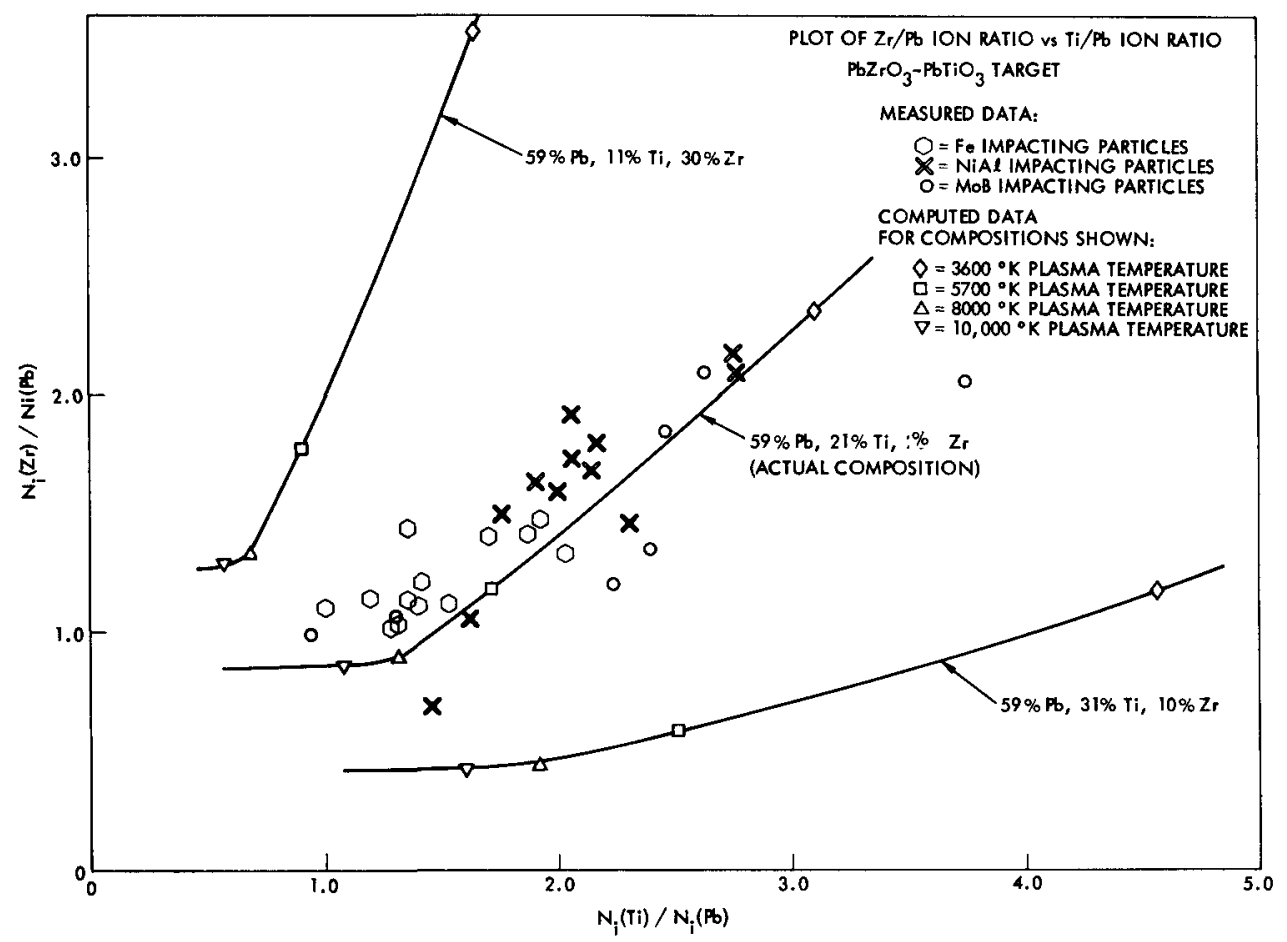

Fraure 3.-Zirconium/lead ion ratio $N_{i}(\mathrm{Zr}) / N_{i}(\mathrm{~Pb})$ vs titanium/lead ion ratio $N_{i}(\mathrm{Ti}) / N_{i}(\mathrm{~Pb})$. Data points are derived from the ratio of the indicated ion groups, the amplitudes of which are measured from the collector waveform. Each point represents a single impact event. The curves shown are computed using the indicated target composition for each and the equations which characterize the impact ionization model. Bending of the curve is caused by the temperature dependence of the partition functions for ions and neutrals of each of the subject elements.

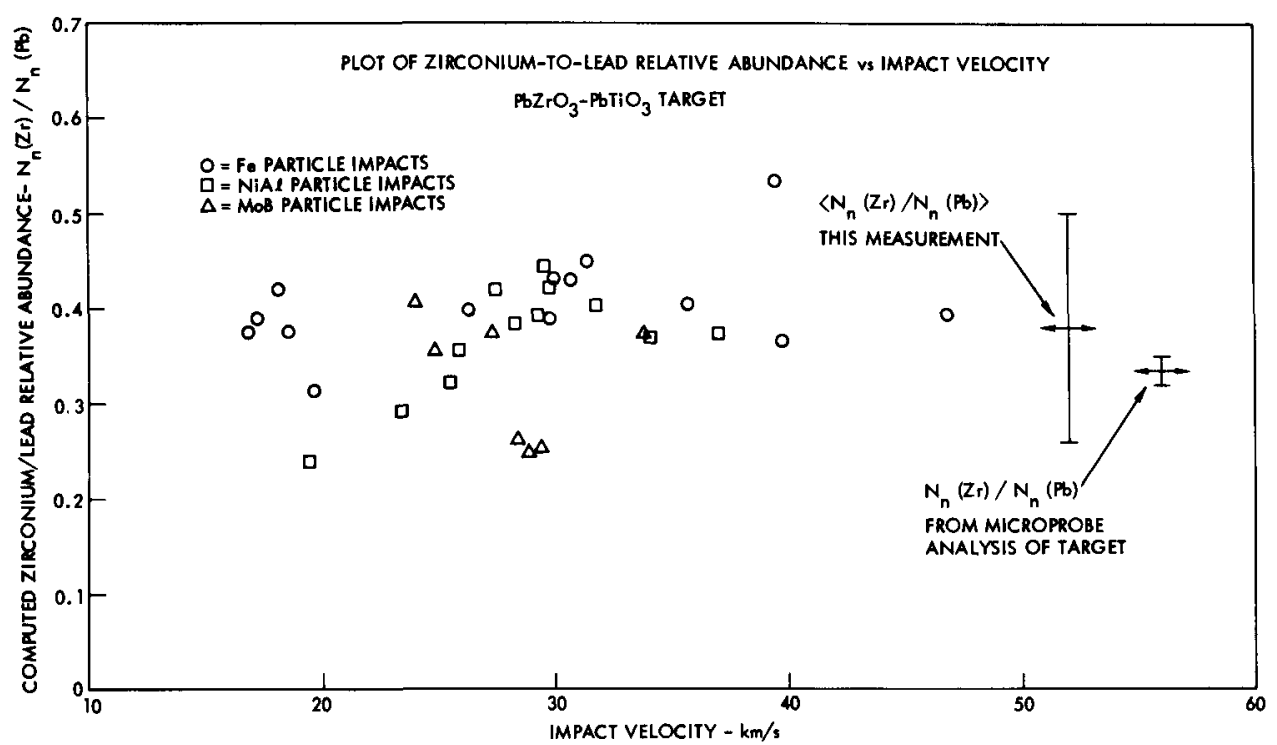


satisfactory two-element particle materials will continue in an effort to answer this question.

Another practical task that must be accomplished is the selection of the control elements in the target. The control elements can, in principle, take several forms; a substrate can by some means be impregnated with the two control elements, a binary compound material can be used, or vapor (or electrolytically) deposited films can be employed. Whatever the form, homogeneity of the materials on a microscopic scale is a necessary condition. Several other factors must also be considered:

(1) The ionization potentials of the two elements must differ by about one electron volt in order to obtain a substantial change in the ratio of the ion signals from the control elements as the plasma temperature changes.
(2) The ionization potentials must be low enough to ensure a sufficient degree of ionization to obtain detectable signals.

(3) The mass of the atoms should lie outside of the mass range of elements expected from the cosmic dust particles.

(4) The materials must be stable when exposed to the space environment.

In conclusion we would suggest that laboratory experimentation for the purposes of further verification (and possible improvement) of the model and selection and test of target materials be continued. An early flight test of even a simplified version of a cosmic dust analyzer would be a highly desirable undertaking. Such a flight test would provide a measure of the signal magnitudes from naturally occurring cosmic dust particles and could crudely identify the major elemental constituents.

\section{REFERENCES}

Aller, L. H., 1963. Astrophysics-The Atmosphere of the Sun and Stars, second edition, The Ronald Press Co., New York, 115-117.

Auer, S., AND SiTTe, K., 1968. Detection technique for micrometeoroids using impact ionization, Earth Planet. Sci. Letters, 4, 178-183.

Frinchtenicht, J. F., 1962. Two-million-volt electrostatic accelerator for hypervelocity research, Rev. Sci. Instr., 33, 209-212.

Fritchtenicht J. F., AND Slattery, J. C., 1963. Ionization associated with hypervelocity impact, NASA Tech. Note D-2091.

Hansen, D. O., 1968. Mass analysis of ions produced by hypervelocity impact, Appl. Phys. Letters, 13, 89-91.

Hansen, D. O., and Roy, N. I., 1966. A solid-state low-noise preamplifier, Nucl. Instr. Methods, 40, 209-212.

Roy, N. L., AND Becker, D. G., 1971. A time interval selector and proportional delay generator, Rev. Sci. Instr., 42, 204-209.

Shelton, H., Hendricks, C. D., Jr., And Wuerker, R. F., 1960. Electrostatic acceleration of microparticles to hypervelocities, J. Appl. Phys., 31, 1243-1246.

Sutton, G. W., And Sherman, A., 1965. Engineering Magnelohydrodynamics, MeGraw-Hill Book Co., New York, 225. 